\title{
Two cases of clostridial hepatitis
}

\author{
DAVID J. B. ASHLEY
}

From the Department of Pathology, Morriston Hospital, Swansea

SYNOPSIS Two cases are described in which clostridial invasion of the liver and spleen was noted at post-mortem examination. In each there was a history of debilitating disease and it is suggested that the infection was consequent upon impaired immunological and cellular defence mechanisms.

Clostridial infection of the liver and spleen with the production of gas-filled cysts is an unusual occurrence even in cases where necropsy has been delayed for many hours after death. Two cases are described in which this phenomenon was observed and in which it was concluded that the viscera had been invaded before death. A few cases have been seen following infections of the biliary tract or operations thereon or as part of a generalized clostridial septicaemia, and Potter (1952) mentions gas production in the livers of infants who have died in utero and been retained there for some days.

\section{CASE HISTORIES}

CASE 1 This patient was a girl who, at the time of her last admission to hospital in 1962, was 17 years old. She was first seen in 1960 when a diagnosis of Hodgkin's disease was made on biopsy of a cervical lymph node. She was treated by deep $x$-ray therapy on two occasions and later was given a course of prednisone and one of nitrogen mustard. At the time of her penultimate discharge from hospital her white cell count was 1,600 per c.mm and her platelet count 53,000 per c.mm. Two weeks later she was admitted for the last time as an emergency. Now she was drowsy and complained of abdominal pain, vomiting, and diarrhoea which had been present for five days. She was pyrexial; the abdomen was distended and tender. No significant localizing signs were noted and laboratory investigations were unhelpful. Her temperature during the five days of her last admission oscillated between $98^{\circ} \mathrm{F}$. and $104^{\circ} \mathrm{F}$. $\left(37^{\circ} \mathrm{C} .-40^{\circ} \mathrm{C}\right.$.), her condition steadily deteriorated and she died on the fifth day. No antibiotics or chemotherapeutic agents were administered during this last period in hospital.

Post-mortem examination A necropsy was carried out four hours after death. The cranium and its contents appeared normal. There was a little blood-stained fluid in the pericardial cavity but the myocardium, endocardium, and pericardium appeared normal. The trachea and main bronchi contained mucopurulent fluid and there was patchy bronchopneumonia in both lungs. A

Received for publication 27 April 1964. small infarct was present at the base of the left lung. The pleural cavities appeared normal. There was an acute purulent peritonitis with many adhesions but no local lesion of the stomach or intestines could be found and there was no evidence of a leak from the gastrointestinal tract. The Fallopian tubes and ovaries showed an acute inflammatory reaction. The spleen was grossly congested and was diffluent. The kidneys were enlarged and pale. The liver was enlarged, pale and fatty, and felt crepitant; on section it proved to contain many gas-filled spaces (Fig. 1). When a portion of the liver was thrown into water it floated.

Histology Sections of the Fallopian tube and ovary showed an inflammatory exudate on the surface with many lymphocytes and plasma cells but few polymorphonuclear leucocytes. The lungs showed an exudate in the alveolar lumina and many colonies of bacilli in the alveoli and blood vessels but no vital reaction. The spleen was congested and showed complete loss of the normal architecture which was replaced by sheets of reticulum cells and, here and there, a few giant cells. A few spaces filled with a protein-containing exudate were seen, and were bordered by compressed splenic tissue. The kidney showed widespread degeneration of the epithelium of the convoluted tubules in the cortex with cloudy swelling of the cytoplasm of the cells and loss of the nuclear pattern and staining reaction.

The liver showed generalized oedema and separation of the parenchymal cells. There were many gas-filled spaces lined by flattened liver cells and showing no vital reaction (Fig. 2). Gram-positive bacilli were present in and between the cells adjacent to the cysts (Fig. 3). There was infiltration of occasional portal tracts by plasma cells and lymphocytes. No evidence of reticulo-endothelial tumour was found in the liver substance.

CASE 2 This patient was a man who, at the time of his death, was 53 years of age. He had been a patient at this hospital since 1960 when he was first admitted because of liver failure, and a diagnosis of haemochromatosis was made. At his last admission in 1963 he was confused and drowsy and his speech was slurred. The abdomen was distended and there was some ankle oedema. Despite treatment he died in hepatic coma. His temperature during the month of his last admission remained normal 


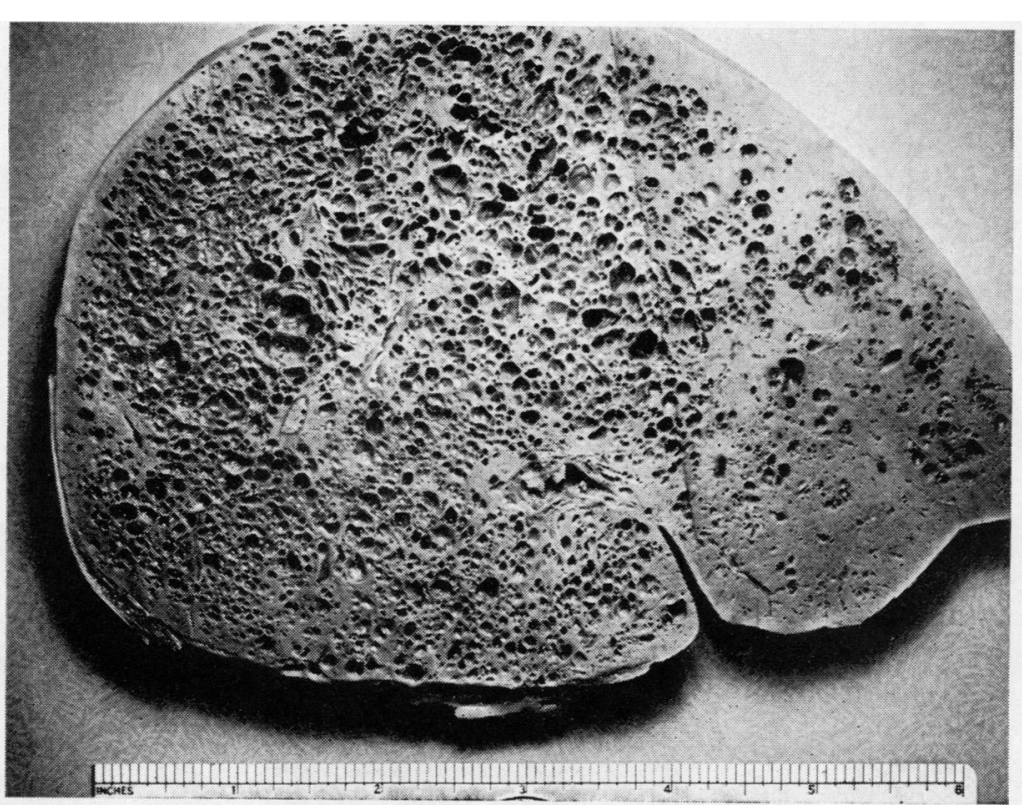

FIG. 1. Case 1: liver showing widespread cystic change.

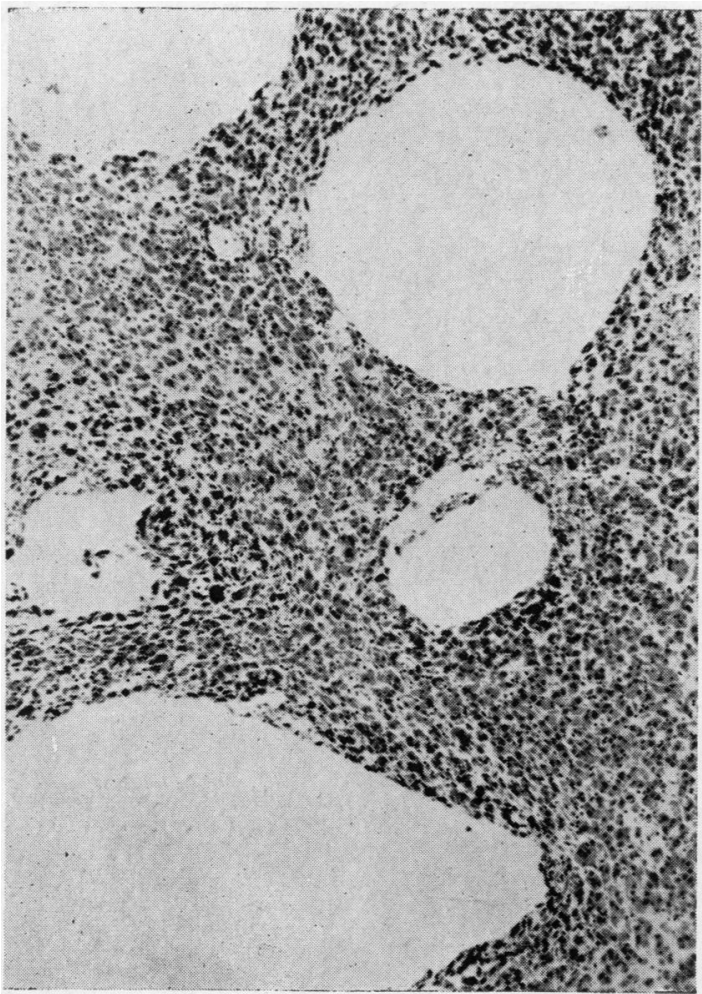

FIG. 2. Case 1: liver showing gas-filled cysts without vital reaction. Haematoxylin and eosin $\times 100$.

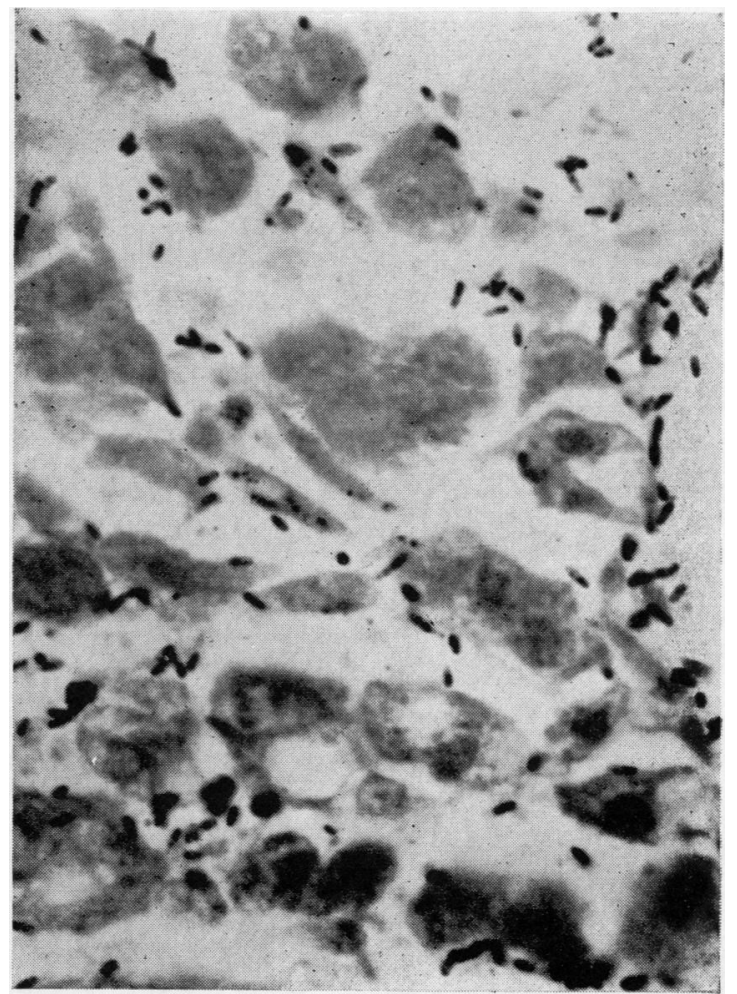

FIG. 3. Case 1: liver showing Gram-positive bacilli in the wall of a cyst. Gram $\times 720$. 


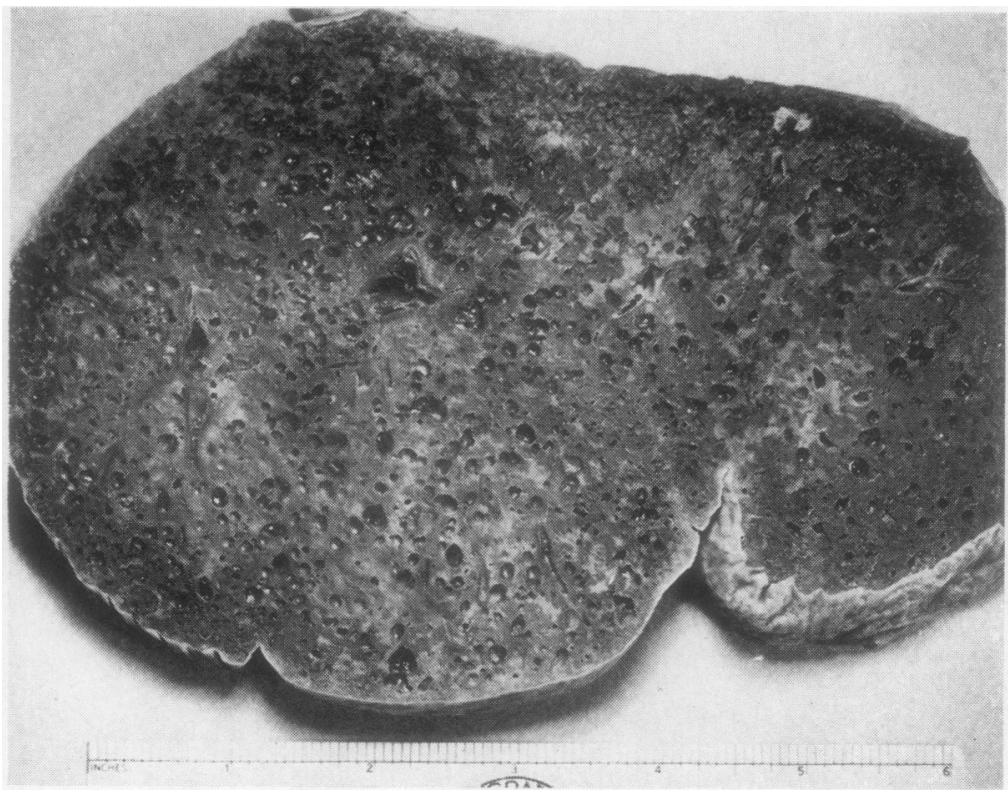

FIG. 4. Case 2: spleen showing multiple cysts.

except for an occasional peak of $99^{\circ} \mathrm{F}$. $\left(37 \cdot 4^{\circ} \mathrm{C}\right.$.) and one of $100^{\circ} \mathrm{F} .\left(37 \cdot 7^{\circ} \mathrm{C}\right.$.) two days before death. Antibiotics were not administered during the last period in hospital. The white blood cells were normal in number.

Post-mortem examination A necropsy was carried out 24 hours after death. There was extensive intravascular haemolysis and the intima of the major vessels, both arteries and veins, was stained with haemoglobin. The trachea and main bronchi contained aspirated bloodstained vomit. Both lungs were oedematous. No varices were apparent in the oesophagus. The gastric mucosa was reddened and had a polypoid appearance. No anomaly was noted in the intestine. The peritoneal cavity contained about 4 pints of amber fluid but no inflammatory reaction was noted.

The liver was small and showed a nodular tawny cut surface with much fibrosis. Many gas-filled spaces were present and a portion of liver floated when placed in water. The pancreas was dark in colour. The spleen was enlarged, the pulp was soft, and there were many gasfilled spaces in it (Fig. 4). Both kidneys were enlarged and pale.

Histology Sections of the lung showed widespread oedema and small patches of bronchopneumonia. There was mild chronic hypertrophic gastritis and the peritoneal surface of the stomach showed a fibrinous exudate in which were many colonies of Gram-positive bacilli. The kidneys showed marked degenerative changes in the cortical convoluted tubules; the glomeruli showed a membranous glomerulo-nephritis. Many colonies of organisms were seen scattered through the renal parenchyma.

The liver was nodular and broad bands of fibrous tissue showing infiltration with plasma cells and lymphocytes criss-crossed the substance of the organ. The centres of the nodules of parenchymal cells showed marked fatty change and many of the liver cells contained brown pigment which, on special staining, proved to be haemosiderin. There were many small cystic spaces surrounded by distorted liver cells (Fig. 5). There was no inflammatory reaction in these areas. Many small ducts were

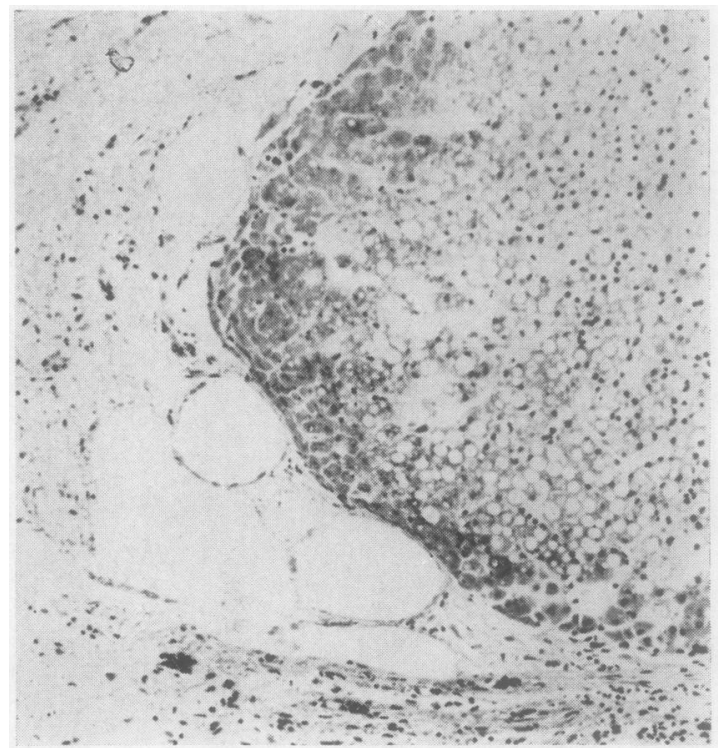

FIG. 5. Case 2: liver showing portal fibrosis, fatty change in the parenchyma, and a cyst in which there is no vital reaction. Haematoxylin and eosin $\times 110$. 
present in the fibrous tissue, some of which contained inspissated bile.

Small cysts were scattered through the parencyhma of the spleen. The walls of the cysts consisted of flattened and distorted splenic tissue.

\section{BACTERIOLOGY}

Specimens were taken from the liver in case 1 and from the liver and spleen in case 2 . In each instance a pure growth of an anaerobic spore-bearing Grampositive bacillus was obtained and in each the identity of the organism was established as Clostridium welchii by the following criteria:-Oval, subterminal spores were present in a relatively large bacillus which was an obligatory anaerobe. The colonies on blood agar were round and smooth and there was a narrow zone of haemolysis. A stormy clot was produced in litmus milk. Lactose, glucose, sucrose, and maltose were fermented and a lethicinase was produced which was specifically inhibited by Clostridium welchii antitoxin.

\section{DISCUSSION}

These two cases appear to be examples of an unusual type of infection. Clostridia may be found in the lumen of the biliary tract in a proportion of patients submitted to operations on these organs (Kivel, Kessler, and Cameron, 1958) and local infection of the liver may ensue either spontaneously (Jarkowski and Wolf, 1962) or after operations in this region (Mackay, Caldwell, and Hindawi, 1963). A case showing some similarity to the first case described in this paper is case 1 of Jarkowski and Wolf, a 21-year-old girl suffering from acute lymphatic leukaemia, who developed clostridial enteritis and septicaemia and was found, at necropsy, to have gas-filled cysts in the liver, spleen, and brain. Clostridial infection of the liver, without septicaemia, is also mentioned by Potter (1952) in the case of stillborn infants.

It is unlikely that the necropsy finding of clostridial infection in these cases can be explained on the hypothesis that the gas-filled spaces were a consequence of post-mortem invasion of the tissues. In case 1 there were abdominal symptoms for some days before death and there was a vital reaction, acute peritonitis, which could only have developed in vivo. It is suggested that this infection was due to a concomitant leakage of other faecal organisms. In this case too the necropsy was carried out only four hours after death, a period which appears too short for widespread dissemination of micro-organisms. In case 2 necropsy was delayed for 24 hours but the body was refrigerated during this interval. In this case no clinical manifestations of ante-mortem infection were apparent. This man was, however, obviously dying during his last few days and the diminishing vital functions of a moribund patient are not conducive to the ready recognition of symptoms which, in his case, in the absence of overt peritonitis, would be expected to be non-specific in nature.

Since the first case was seen the possibility of post-mortem spread of gas-forming organisms has naturally been very much in mind. My colleague, Dr. H. Duncan Davies, and I have examined all livers carefully for signs of invasion by gas-forming organisms and, even in the necropsies carried out in public mortuaries where the cadaver had been kept at room temperature for two or three days, have failed to find any evidence that post-mortem invasion of the tissues by clostridia has resulted in the formation of gas-filled cysts in the organs.

The alternative explanation is that in both instances infection of the liver and other organs occurred before death. In case 1 there were clinical signs of an abdominal illness and at necropsy evidence of peritonitis was found in addition to the local changes in the liver. This patient had suffered for two years from a tumour of the reticulo-endothelial system, Hodgkin's disease. She had been treated by radiotherapy, with an adreno-cortical steroid hormone, prednisone, and with a radiomimetic drug, nitrogen mustard. Her terminal illness can easily, in retrospect, be diagnosed as peritonitis and portal septicaemia due to leakage of intestinal organisms into the peritoneal cavity where they produced peritonitis and into the portal blood stream whence they travelled to the liver and spleen and there produced the clostridial hepatitis.

In the second case there was also a long-standing illness which had necessitated the patient's admission to hospital on several occasions and which was of widespread nature in that a number of organs were affected. In this case the spleen was enlarged, presumably as a consequence of portal hypertension, itself due to the cirrhosis hepatis, and the development of gas cysts was more prominent in this organ than in the liver.

In both cases other organs were invaded by the bacilli and in each there was degenerative change in the convoluted tubules of the kidneys, a toxic phenomenon related to the severe illness from which each was suffering, and also possibly to the effects of toxic substances liberated by the invading bacteria. The absence of vital reaction of infiltration of the affected organs by inflammatory cells is not itself evidence of a post-mortem origin of the infection. Both patients were debilitated and the girl (case 1) was suffering from a disease of the erticulo-endo- 
thelial system which had resulted in a reduction in the number of circulating white blood cells. Furthermore, in severe cases of gas gangrene of the classical type there is not usually any local evidence of inflammatory infiltration (Anderson, 1961).

It is probable that organisms can escape from the inside of the intestine to the portal circulation, as they do in the transient bacteraemia known to follow dental extraction, but that normally the defence mechanism of the body is adequate to destroy the invading microbes. In the present two cases the defences of the body were impaired, in the first case because of reticulo-endothelial disease and treatment with therapeutic agents known to have the side-effect of reducing immunological reactions, and in the second because of long-standing metabolic disease. It is suggested that the unusual phenomena observed in these two cases are to be attributed to incapacity of the defence mechanism in the face of minor bacterial invasion. A similar mechanism may be invoked in the patient of Jarkowski and Wolf (1962) who had been suffering from acute lymphatic leukaemia for some months before death.

I am indebted to Dr. E. A. Danino for the clinical data on these patients who were admitted under his care.

\section{REFERENCES}

Anderson, W. A. D. (1961). Pathology, 4th ed., p. 225. Mosby, St. Louis.

Jarkowski, T. L., and Wolf, P. L. (1962). J. Amer. med. Ass., 181, 845. Kivel, R. M., Kessler, A., and Cameron, D. J. (1958). Ann. intern. Med., 49, 672.

Mackay, A. G., Caldwell, E. J., and Hindawi, R. K. (1963). New Engl. J. Med., 268, 534.

Potter, E. L. (1952). Pathology of the Fetus and the Newborn, p. 332. Year Book Publishers, Chicago. 